\title{
Few-Shot and Zero-Shot Multi-Label Learning for Structured Label Spaces
}

\author{
Anthony Rios \\ Department of Computer Science \\ University of Kentucky \\ Lexington, KY \\ anthony.riosl@uky . edu
}

\author{
Ramakanth Kavuluru \\ Division of Biomedical Informatics \\ University of Kentucky \\ Lexington, $\mathrm{KY}$ \\ ramakanth.kavuluru@uky . edu
}

\begin{abstract}
Large multi-label datasets contain labels that occur thousands of times (frequent group), those that occur only a few times (few-shot group), and labels that never appear in the training dataset (zero-shot group). Multi-label few- and zero-shot label prediction is mostly unexplored on datasets with large label spaces, especially for text classification. In this paper, we perform a fine-grained evaluation to understand how state-of-the-art methods perform on infrequent labels. Furthermore, we develop few- and zero-shot methods for multilabel text classification when there is a known structure over the label space, and evaluate them on two publicly available medical text datasets: MIMIC II and MIMIC III. For fewshot labels we achieve improvements of $6.2 \%$ and 4.8\% in R@ 10 for MIMIC II and MIMIC III, respectively, over prior efforts; the corresponding R@10 improvements for zero-shot labels are $17.3 \%$ and $19 \%$.
\end{abstract}

\section{Introduction}

Unlike in binary or multi-class problems, for multi-label classification a model assigns a set of labels to each input instance (Tsoumakas et al., 2010). Large-scale multi-label text classification problems can be found in several domains. For example, Wikipedia articles are annotated with labels used to organize documents and facilitate search (Partalas et al., 2015). Biomedical articles indexed by the PubMed search engine are manually annotated with medical subject headings (Tsatsaronis et al., 2012). In healthcare facilities, medical records are assigned a set of standardized codes for billing purposes (NCHS, 1978). Automatically annotating tweets with hashtags, while the labels are not fixed, can also be represented as a large-scale multi-label classification problem (Weston et al., 2014).

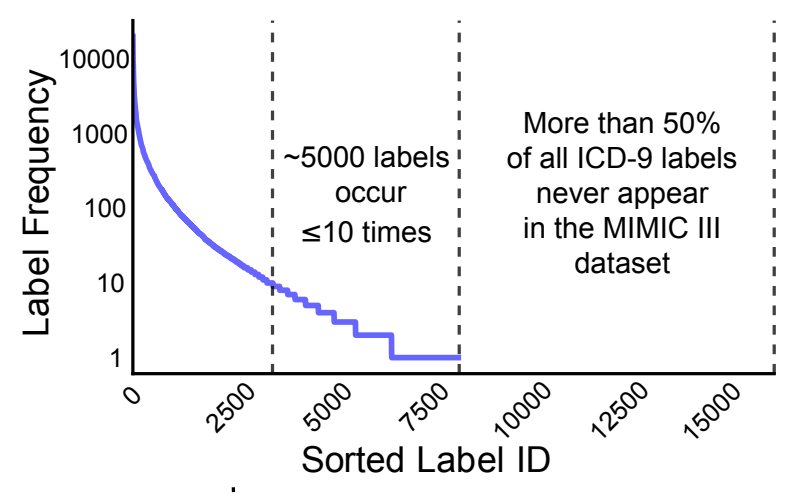

Figure 1: This plot shows the label frequency distribution of ICD-9 codes in MIMIC III.

There are two major difficulties when developing machine learning methods for large-scale multi-label text classification problems. First, the documents may be long, sometimes containing more than a thousand words (Mullenbach et al., 2018). Finding the relevant information in a large document for a specific label results in needle in a haystack situation. Second, data sparsity is a common problem; as the total number of labels grows, a few labels may occur frequently, but most labels will occur infrequently. Rubin et al. (2012) refer to datasets that have long-tail frequency distributions as "power-law datasets". Methods that predict infrequent labels fall under the paradigm of few-shot classification which refers to supervised methods in which only a few examples, typically between 1 and 5, are available in the training dataset for each label. With predefined label spaces, some labels may never appear in the training dataset. Zeroshot problems extend the idea of few-shot classification by assuming no training data is available for the labels we wish to predict at test time. In this paper, we explore both of these issues, long documents and power-law datasets, with an emphasis on analyzing the few- and zero-shot aspects of large-scale multi-label problems. 
In Figure 1, we plot the label frequency distribution of diagnosis and procedure labels for the entire MIMIC III (Johnson et al., 2016) dataset. A few labels occur more than 10,000 times, around 5,000 labels occur between 1 and 10 times, and of the 17,000 diagnosis and procedure labels, more than $50 \%$ never occur. There are a few reasons a label may never occur in the training dataset. In healthcare, sevearl disorders are rare; therefore corresponding labels may not have been observed yet in a particular clinic. Sometimes new labels may be introduced as the field evolves leading to an emerging label problem. This is intuitive for applications such as hashtag prediction on Twitter. For example, last year it would not have made sense to annotate tweets with the hashtag \#EMNLP2018. Yet, as this year's conference approaches, labeling tweets with the \#EMNLP2018 will help users find relevant information.

Infrequent labels may not contribute heavily to the overall accuracy of a multi-label model, but in some cases, correct prediction of such labels is crucial but not straightforward. For example, in assigning diagnosis labels to EMRs, it is important that trained human coders are both accurate and thorough. Errors may cause unfair financial burden on the patient. Coders may have an easier time assigning frequent labels to EMRs because they are encountered more often. Also, frequent labels are generally easier to predict using machine-learning based methods. However, infrequent or obscure labels will be easily confused or missed causing billing mistakes and/or causing the coders to spend more time annotating each record. Thus, we believe methods that handle infrequent and unseen labels in the multi-label setting are important.

Current evaluation methods for large-scale multi-label classification mostly ignore infrequent and unseen labels. Popular evaluation measures focus on metrics such as micro-F1, recall at $\mathrm{k}(\mathrm{R} @ \mathrm{k})$, precision at $\mathrm{k}(\mathrm{P} @ \mathrm{k})$, and macro-F1. As it is well-known that micro-F1 gives more weight to frequent labels, papers on this topic also report macro-F1, the average of label-wise F1 scores, which equally weights all labels. Unfortunately, macro-F1 scores are generally low and the corresponding performance differences between methods are small. Moreover, it is possible to improve macro-F1 by only improving a model's performance on frequent labels, further confounding its interpretation. Hence we posit that macro-F1 is not enough to compare large-scale multi-label learning methods on infrequent labels and it does not directly evaluate zero-shot labels. Here, we take a step back and ask: can the model predict the correct few-shot (zero-shot) labels from the set of all few-shot (zero-shot) labels? To address this, we test our approach by adapting the generalized zero-shot classification evaluation methodology by Xian et al. (2017) to the multi-label setting.

In this paper, we propose and evaluate a neural architecture suitable for handling few- and zeroshot labels in the multi-label setting where the output label space satisfies two constraints: (1). the labels are connected forming a DAG and (2). each label has a brief natural language descriptor. These assumptions hold in several multi-label scenarios including assigning diagnoses/procedures to EMRs, indexing biomedical articles with medical subject headings, and patent classification. Taking advantage of this prior knowledge on labels is vital for zero-shot prediction. Specifically, using the EMR coding use-case, we make the following contributions:

1. We overcome issues arising from processing long documents by introducing a new neural architecture that expands on recent attentionbased CNNs (ACNNs (Mullenbach et al., 2018)). Our model learns to predict few- and zero-shot labels by matching discharge summaries in EMRs to feature vectors for each label obtained by exploiting structured label spaces with graph CNNs (GCNNs (Kipf and Welling, 2017)).

2. We provide a fine-grained evaluation of stateof-the-art EMR coding methods for frequent, few-shot, and zero-shot labels. By evaluating power-law datasets using an extended generalized zero-shot methodology that also includes few-shot labels, we present a nuanced analysis of model performance on infrequent labels.

\section{Related Work}

Large-Scale Text Classification. Linear methods have been successfully applied to large-scale problems (Tang et al., 2009; Papanikolaou et al., 2015; Rios and Kavuluru, 2015). For traditional micro- and macro-F1 measures, Tang et al. (2009) show that linear methods suffer using naive thresh- 
olding strategies because infrequent labels generally need a smaller threshold. Generative models have also been promising for datasets with many labels (Rubin et al., 2012). Intuitively, by using a prior distribution over the label space, infrequent labels can be modeled better. Finally, large-scale classification is also pursued as "extreme classification" (Yu et al., 2014; Bhatia et al., 2015) where the focus is on ranking measures that ignore infrequent labels. Neural networks (NNs) perform well for many small-scale classification tasks (Kim, 2014; Kalchbrenner et al., 2014). Recently, researchers have been exploring NN methods for large-scale problems. Yang et al. (2016) develop a hierarchical attentive NN for datasets with over a million documents, but their datasets contain few labels. Nam et al. (2014) show that feed-forward NNs can be successfully applied to large-scale problems through the use of a multilabel binary cross-entropy loss function. Vani et al. (2017) introduce a grounded recurrent neural network (RNN) that iteratively updates its predictions as it processes a document word-by-word. Baumel et al. (2018) experiment with both CNNs and RNNs for medical coding. Finally, Mullenbach et al. (2018) expand on prior ACNNs (Yang et al., 2016; Allamanis et al., 2016) to develop a labelwise attention framework where the most informative ngrams are extracted for each label in the dataset. Our attention mechanism extends their work to the zero-shot setting.

Few-Shot and Zero-Shot Learning. While neural networks are generally considered to need large datasets, they have been shown to work well on few-shot classification tasks. To handle infrequent labels, most NN methods use a $k-\mathrm{NN}$ like approach. Siamese NNs (Koch et al., 2015) learn a nonlinear distance metric using a pairwise loss function. Matching networks (Vinyals et al., 2016) introduce an instance-level attention method to find relevant neighbors. Prototypical Networks (Snell et al., 2017) average all instances in each class to form "prototype label vectors" and train using a traditional cross-entropy loss. In our prior work (Rios and Kavuluru, 2018), we combine matching networks with a sophisticated thresholding strategy. However, in Rios and Kavuluru (2018) we did not explore the few- and zeroshot settings.

Zero-shot learning has not been widely explored in the large-scale multi-label classification scenario. Like neural few-shot methods, neural zero-shot methods use a matching framework. Instead of matching input instances with other instances, they are matched to predefined label vectors. For example, the Attributes and Animals Dataset (Xian et al., 2017) contains images of animals and the label vectors consist of features describing the types of animals (e.g., stripes: yes). When feature vectors for labels are not available, the average of the pretrained word embeddings of the class names have been used. The attribute label embedding method (Akata et al., 2016) uses a pairwise ranking loss to match zero-shot label vectors to instances. Romera-Paredes and Torr (2015) introduced the "embarrassingly simple zero-shot learning" (ESZSL) method which is trained using a mean squared error loss. A few zero-shot methods do not translate well to multi-label problems. CONSE (Mikolov et al., 2013) averages the embeddings for the top predicted supervised label vectors to match to zero-shot label vectors. CONSE assumes that both supervised and zeroshot labels cannot be assigned to the same instance. In this paper, we expand on the generalized zero-shot evaluation methodology introduced by Xian et al. (2017) to large-scale multilabel classification. Finally, it is important to note that zero-shot classification has been previously studied in the multi-label setting (Mensink et al., 2014). However, they focus on image classification and use datasets with around 300 labels.

Graph Convolutional Neural Networks. GCNNs generalize CNNs beyond $2 \mathrm{~d}$ and $1 \mathrm{~d}$ spaces. Defferrard et al. (2016) developed spectral methods to perform efficient graph convolutions. Kipf and Welling (2017) assume a graph structure is known over input instances and apply GCNNs for semi-supervised learning. GCNNs are applied to relational data (e.g., link prediction) by Schlichtkrull et al. (2018). GCNNs have also had success in other NLP tasks such as semantic role labeling (Marcheggiani and Titov, 2017), dependency parsing (Strubell and McCallum, 2017), and machine translation (Bastings et al., 2017).

There are three GCNN papers that share similarities with our work. (i) Peng et al. (2018) use a GCNN on a word co-occurrence graph for large-scale text classification where the GCNN operates on documents/words, while our GCNN operates on the labels. (ii) Chen et al. (2017) use GCNNs on structured label spaces. However, their 


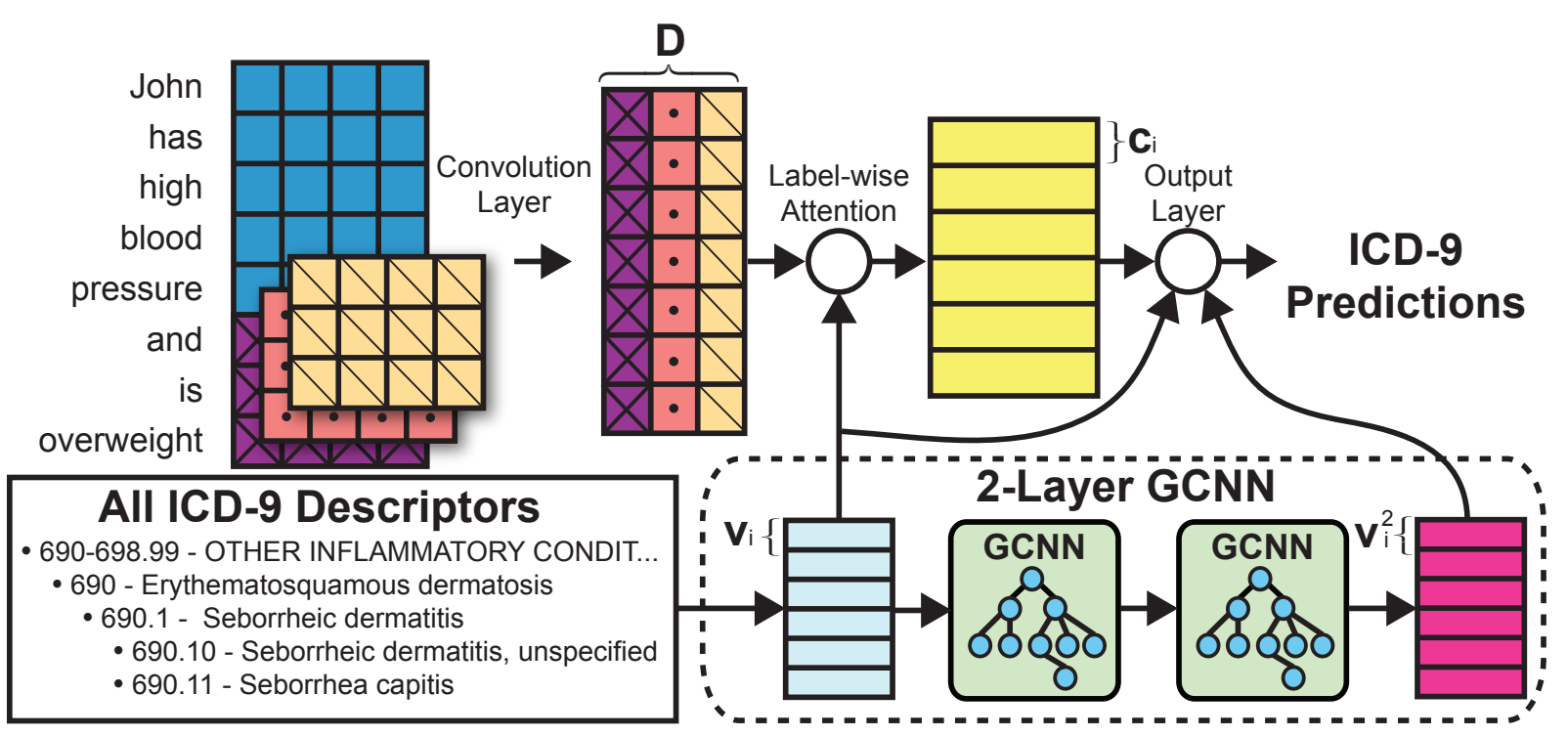

Figure 2: This figure provides a visual overview of our method. Intuitively, our method has two main components. The first component is a CNN that operates operates on the EMRs. The other component is a 2-layer GCNN which creates the label-specific attention vectors and label-vectors used for ranking using ICD-9 descriptions as input.

experiments focus on smaller label spaces and do not handle/assess zero-shot and few-shot labels. Also, their experiments for text classification do not incorporate attention and simply use an average of word vectors to represent each document. (iii) Wang et al. (2018) propose a zero-shot GCNN image classification method for structured multi-class problems. We believe their method may transfer to the multi-label text classification setting but exact modifications to affect that are not clear (i.e., their semi-supervised approach may not be directly applicable). Likewise, porting to text is nontrivial for long documents.

\section{Method}

Figure 2 shows the overall schematic of our architecture. Intuitively, we incorporate four main components. First, we assume we have the full English descriptor/gloss for each label we want to predict. We form a vector representation for each label by averaging the word embeddings for each word in its descriptor. Second, the label vectors formed from the descriptor are used as attention vectors (label-wise attention) to find the most informative ngrams in the document for each label. For each label, this will produce a separate vector representation of the input document. Third, the label vectors are passed through a two layer GCNN to incorporate hierarchical information about the label space. Finally, the vectors returned from the GCNN are matched to the document vectors to generate predictions.

Convolutional Neural Network. Contrary to prior CNN methods for text (Kim, 2014), instead of using a max-over-time pooling layer, we learn to find relevant ngrams in a document for each label via label-wise attention (Mullenbach et al., 2018). The CNN will return a document feature matrix $\mathbf{D} \in \mathbb{R}^{(n-s+1) \times u}$ where each column of $\mathbf{D}$ is a feature map, $u$ is the total number of convolution filters, $n$ is the number of words in the document, and $s$ is the width of convolution filters.

Label Vectors. To be able to predict labels that were not in the training dataset, we avoid learning label specific parameters. We use the label descriptors to generate a feature vector for each label. First, to preprocess each descriptor, we lowercase all words and remove stop-words. Next, each label vector is formed by averaging the remaining words in the descriptor

$$
\mathbf{v}_{i}=\frac{1}{|N|} \sum_{j \in N} \mathbf{w}_{j}, \quad i=1, \ldots, L
$$

where $\mathbf{v}_{i} \in \mathbb{R}^{d}, L$ is the number of labels, and $N$ is the index set of the words in the descriptor. Prior zero-shot work has focused on projecting input instances into the same semantic space as the label vectors (Sandouk and Chen, 2016). For zero-shot image classification, this is a non-trivial task. Because we work with textual data, we simply share 
the word embeddings between the convolutional layer and the label vector creation step to form $\mathbf{v}_{i}$.

Label-Wise Attention. Similar to the work by Mullenbach et al. (2018), we employ label-wise attention to avoid the needle in the haystack situation encountered with long documents. The issue with simply using a single attention vector or using max-pooling is that we assume a single vector can capture everything required to predict every label. For example, with a single attention, we would only look at one spot in the document and assume that spot contains the relevant information needed to predict all labels. In the multi-class setting, this assumption is plausible. However, for large multilabel problems, the relevant information for each label may be scattered throughout the document - the problem is worse when the documents are very long. Using label-wise attention, our model can focus on different sections. We also need to find relevant information for zero-shot classes. So we use the label vectors $\mathbf{v}_{i}$ rather than learning label specific attention parameters. First, we pass the document feature matrix $\mathbf{D}$ through a simple feed-forward neural network

$$
\mathbf{D}^{2}=\tanh \left(\mathbf{D} \mathbf{W}_{b}+\mathbf{b}_{b}\right)
$$

where $\mathbf{W}_{b} \in \mathbb{R}^{u \times d}$ and $\mathbf{b}_{b} \in \mathbb{R}^{d}$. This mapping is important because the dimensionality of the ngram vectors (rows) in $\mathbf{D}$ depends on $u$, the number of scores we generate for each ngram. Given $\mathbf{D}^{2}$, we generate the label-wise attention vector

$$
\mathbf{a}_{i}=\operatorname{softmax}\left(\mathbf{D}^{2} \mathbf{v}_{i}\right), \quad i=1, \ldots, L,
$$

where $\mathbf{a}_{i} \in \mathbb{R}^{n-s+1}$ measures how informative each ngram is for the $i$-th label. Finally, we use $\mathbf{D}$, and generate $L$ label-specific document vector representations

$$
\mathbf{c}_{i}=\mathbf{a}_{i}^{T} \mathbf{D}, \quad i=1, \ldots, L,
$$

such that $\mathbf{c}_{i} \in \mathbb{R}^{u}$. Intuitively, $\mathbf{c}_{i}$ is the weighted average of the rows in $\mathbf{D}$ forming a vector representation of the document for the $i$-th label.

GCNN Output Layer. Traditionally, the output layer of a CNN would learn label specific parameters optimized via a cross-entropy loss. Instead, our method attempts to match documents to their corresponding label vectors. In essence, this becomes a retrieval problem. Before using each document representation $\mathbf{c}_{i}$ to score its corresponding label, we take advantage of the structured knowledge we have over our label space using a 2-layer GCNN. For both the MIMIC II and MIMIC III datasets, this information is hierarchical. A snippet of the hierarchy can be found in Figure 2.

Starting with the label vectors $\mathbf{v}_{i}$, we combine the label vectors of the children and parents for the $i$-th label to form

$\mathbf{v}_{i}^{1}=f\left(\mathbf{W}^{1} \mathbf{v}_{i}+\sum_{j \in \mathcal{N}_{p}} \frac{\mathbf{W}_{p}^{1} \mathbf{v}_{j}}{\left|\mathcal{N}_{p}\right|}+\sum_{j \in \mathcal{N}_{c}} \frac{\mathbf{W}_{c}^{1} \mathbf{v}_{j}}{\left|\mathcal{N}_{c}\right|}+\mathbf{b}_{g}^{1}\right)$ where $\mathbf{W}^{1} \in \mathbb{R}^{q \times d}, \mathbf{W}_{p}^{1} \in \mathbb{R}^{q \times d}, \mathbf{W}_{c}^{1} \in \mathbb{R}^{q \times d}$, $\mathbf{b}_{g}^{1} \in \mathbb{R}^{q}, f$ is the rectified linear unit (Nair and Hinton, 2010) function, and $\mathcal{N}_{c}\left(\mathcal{N}_{p}\right)$ is the index set of the $i$-th label's children (parents). We use different parameters to distinguish each edge type. In this paper, given we only deal with hierarchies, the edge types include edges from parents, from children, and self edges. This can be adapted to arbitrary DAGs, where parent edges represent all incoming edges and the child edges represent all outgoing edges for each node.

The second layer follows the same formulation as the first layer with

$\mathbf{v}_{i}^{2}=f\left(\mathbf{W}^{2} \mathbf{v}_{i}^{1}+\sum_{j \in \mathcal{N}_{p}} \frac{\mathbf{W}_{p}^{2} \mathbf{v}_{j}^{1}}{\left|\mathcal{N}_{p}\right|}+\sum_{j \in \mathcal{N}_{c}} \frac{\mathbf{W}_{c}^{2} \mathbf{v}_{j}^{1}}{\left|\mathcal{N}_{c}\right|}+\mathbf{b}_{g}^{2}\right)$

where $\mathbf{W}^{2} \in \mathbb{R}^{q \times q}, \mathbf{W}_{p}^{2} \in \mathbb{R}^{q \times q}, \mathbf{W}_{c}^{2} \in \mathbb{R}^{q \times q}$, and $\mathbf{b}_{g}^{2} \in \mathbb{R}^{q}$. Next, we concatenate both the averaged description vector (from equation (1)) with the GCNN label vector to form

$$
\mathbf{v}_{i}^{3}=\mathbf{v}_{i} \| \mathbf{v}_{i}^{2},
$$

where $\mathbf{v}_{i}^{3} \in \mathbb{R}^{d+q}$. Now, to compare the final label vector $\mathbf{v}_{i}^{3}$ with its document vector $\mathbf{c}_{i}$, we transform the document vector into

$$
\mathbf{e}_{i}=\operatorname{Re} L U\left(\mathbf{W}_{o} \mathbf{c}_{i}+\mathbf{b}_{o}\right), \quad i=1, \ldots, L,
$$

where $\mathbf{W}_{o} \in \mathbb{R}^{(q+d) \times u}$ and $\mathbf{b}_{o} \in \mathbb{R}^{q+d}$. This transformation is required to match the dimension to that of $\mathbf{v}_{i}^{3}$. Finally, the prediction for each label $i$ is generated via

$$
\hat{y}_{i}=\operatorname{sigmoid}\left(\mathbf{e}_{i}^{T} \mathbf{v}_{i}^{3}\right), \quad i=1, \ldots, L .
$$

During experiments, we found that using either the output layer GCNN or a separate GCNN for the attention vectors (equation (2)) did not result in an improvement and severely slowed convergence. 
Training. We train our model using a multilabel binary cross-entropy loss (Nam et al., 2014)

$$
\mathcal{L}=\sum_{i=1}^{L}\left[-y_{i} \log \left(\hat{y}_{i}\right)-\left(1-y_{i}\right) \log \left(1-\hat{y}_{i}\right)\right],
$$

where $y_{i} \in\{0,1\}$ is the ground truth for the $i$-th label and $\hat{y}_{i}$ is our sigmoid score for the $i$-th label.

\section{Experiments}

In this paper, we use two medical datasets for evaluation purposes: MIMIC II (Jouhet et al., 2012) and MIMIC III (Johnson et al., 2016). Both datasets contain discharge summaries annotated with a set of ICD-9 diagnosis and procedure labels. Discharge summaries are textual documents consisting of, but not limited to, physician descriptions of procedures performed, diagnoses made, the patient's medical history, and discharge instructions. Following a generalized zeroshot learning evaluation methodology (Xian et al., 2017), we split the ICD-9 labels into three groups based on frequencies in the training dataset: The frequent group $S$ that contains all labels that occur $>5$ times, the few-shot group $F$ that contains labels that occur between 1 and 5 times, and the zero-shot group $Z$ of labels that never occur in the training dataset, but occur in the test/dev sets. The groups are only used for evaluation. That is, during training, systems are optimized over all labels simultaneously. Instances that do not contain fewor zero-shot classes are removed from their respective groups during evaluation. This grouping is important to assess how each model performs across labels grouped by label frequency. Our evaluation methodology differs from that of Xian et al. (2017) in two ways. First, because each instance is labeled with multiple labels, the same instance can appear in all groups - S, F, and Z. Second, instead of top-1 accuracy or HIT@k evaluation measures, we focus on R@k to handle multiple labels. At a high level, we want to examine whether a model can distinguish the correct fewshot (zero-shot) labels from the set of all few-shot (zero-shot) labels. Therefore, the R@k measures in Tables 2 and 3, and Figure 3 are computed relative to each group.

Evaluation Measures. The overall statistics for these two datasets are reported in Table 1. For reproducibility purposes, we use the same training/test splits of the MIMIC II as Perotte et al.

\begin{tabular}{llllll}
\hline & & & \multicolumn{3}{c}{ \# Labels } \\
Dataset & \# Train & \# Test & S & F & Z \\
\hline MIMIC II & 18822 & 1711 & 3228 & 3459 & 355 \\
MIMIC III & 37016 & 1356 & 4403 & 4349 & 178 \\
\hline
\end{tabular}

Table 1: Dataset statistics for MIMIC II and MIMIC III.

(2013). Following the procedures in Perotte et al. (2013) and Vani et al. (2017), for each diagnosis and procedure label assigned to each medical report, we add its parents using the ICD-9 hierarchy. Each report in MIMIC II is annotated with nearly 37 labels on average using hierarchical label expansion.

MIMIC III does not contain a standardized training/test split. Therefore, we create our own split that ensures the same patient does not appear in both the training and test datasets. Unlike the MIMIC II dataset, we do not augment the labels using the ICD-9 hierarchy. The ICD-9 hierarchy has three main levels. For MIMIC III, level 0 labels make up about $5 \%$ of all occurrences, level 1 labels make up about 62\%, and level 2 (leaf level) labels make up about 33\%. Also, each MIMIC III instance contains16 ICD-9 labels on average.

ICD-9 Structure and Descriptors. The International Classification of Diseases (ICD) contains alphanumeric diagnosis and procedure codes that are used by hospitals to standardize their billing practices. In the following experiments, we use the 9th edition of the ICD ${ }^{1}$. Each ICD-9 identifier contains between 3 to 5 alphanumeric characters of the form $a b c . x y$. The alphanumeric structure defines a simple hierarchy over all ICD-9 codes. For example, "systolic heart failure" (428.2) and "diastolic heart failure" (428.3) are both children of the "heart failure" code 428. Furthermore, sequential codes are grouped together. For instance, numeric codes in the range 390-459 contain "Diseases of the Circulatory System". Furthermore, each code, including groups of codes (390-459), contain short descriptors, where the average descriptor length contains seven words ${ }^{2}$. In this work, we use both the group descriptors and in-

\footnotetext{
${ }^{1}$ The US transitioned from ICD-9 to ICD-10 in 2015. Unfortunately, at the time of publication, large publicly available ICD-10 EMR datasets are unavailable.

${ }^{2}$ The descriptors and hierarchy used in this paper can be found at https://bioportal.bioontology.org/ ontologies/ICD9CM
} 


\begin{tabular}{|c|c|c|c|c|c|c|c|c|}
\hline & \multicolumn{2}{|c|}{$\mathrm{S}$} & \multicolumn{2}{|c|}{$\mathrm{F}$} & \multicolumn{2}{|c|}{$\mathrm{Z}$} & \multicolumn{2}{|c|}{ Harmonic Average } \\
\hline & $\mathrm{R} @ 5$ & $\mathrm{R} @ 10$ & $\mathrm{R} @ 5$ & R@10 & R@5 & R@10 & R@5 & $\mathrm{R} @ 10$ \\
\hline Random & 0.000 & 0.000 & 0.000 & 0.000 & 0.011 & 0.032 & 0.000 & 0.000 \\
\hline Logistic (Vani et al., 2017) * & 0.137 & 0.247 & 0.001 & 0.003 & - & - & - & - \\
\hline CNN (Baumel et al., 2018) * & 0.138 & 0.250 & 0.050 & 0.082 & - & - & - & - \\
\hline ACNN (Mullenbach et al., 2018) * & 0.138 & 0.255 & 0.046 & 0.081 & - & - & - & - \\
\hline Match-CNN (Rios and Kavuluru, 2018) & 0.137 & 0.247 & 0.031 & 0.042 & - & - & - & - \\
\hline ESZSL + W2V & 0.074 & 0.119 & 0.008 & 0.017 & 0.080 & 0.172 & 0.020 & 0.041 \\
\hline ESZSL + W2V 2 & 0.050 & 0.086 & 0.025 & 0.044 & 0.103 & 0.189 & 0.043 & 0.076 \\
\hline ESZSL + GRALS & 0.135 & 0.238 & 0.081 & 0.123 & 0.085 & 0.136 & 0.095 & 0.152 \\
\hline ZACNN & 0.135 & 0.245 & 0.103 & 0.149 & 0.147 & 0.221 & 0.128 & 0.205 \\
\hline ZAGCNN & 0.135 & 0.247 & 0.130 & 0.185 & 0.269 & 0.362 & 0.160 & 0.246 \\
\hline
\end{tabular}

Table 2: MIMIC II results across frequent (S), few-shot (F), and zero-shot (Z) groups. We mark prior methods for MIMIC datasets that we implemented with a*.

\begin{tabular}{|c|c|c|c|c|c|c|c|c|}
\hline & \multicolumn{2}{|c|}{$\mathrm{S}$} & \multicolumn{2}{|c|}{$\mathrm{F}$} & \multicolumn{2}{|c|}{$\mathrm{Z}$} & \multicolumn{2}{|c|}{ Harmonic Average } \\
\hline & $\mathrm{R} @ 5$ & R@ 10 & R@5 & R@10 & $\mathrm{R} @ 5$ & R@10 & $\mathrm{R} @ 5$ & R@10 \\
\hline Random & 0.000 & 0.000 & 0.000 & 0.000 & 0.038 & 0.052 & 0.000 & 0.000 \\
\hline Logistic (Vani et al., 2017) * & 0.273 & 0.427 & 0.014 & 0.014 & - & - & - & - \\
\hline CNN (Baumel et al., 2018) * & 0.269 & 0.413 & 0.058 & 0.085 & - & - & - & - \\
\hline ACNN (Mullenbach et al., 2018) * & 0.288 & 0.458 & 0.130 & 0.168 & - & - & - & - \\
\hline Match-CNN (Rios and Kavuluru, 2018) & 0.278 & 0.426 & 0.049 & 0.060 & - & - & - & - \\
\hline ESZSL + W2V & 0.135 & 0.191 & 0.031 & 0.051 & 0.157 & 0.257 & 0.065 & 0.105 \\
\hline $\mathrm{ESZSL}+\mathrm{W} 2 \mathrm{~V} 2$ & 0.127 & 0.189 & 0.031 & 0.048 & 0.148 & 0.305 & 0.063 & 0.102 \\
\hline ESZSL + GRALS & 0.256 & 0.393 & 0.033 & 0.060 & 0.076 & 0.138 & 0.064 & 0.114 \\
\hline ZACNN & 0.278 & 0.435 & 0.152 & 0.195 & 0.364 & 0.442 & 0.232 & 0.310 \\
\hline ZAGCNN & 0.283 & 0.445 & 0.166 & 0.216 & 0.428 & 0.495 & 0.252 & 0.337 \\
\hline
\end{tabular}

Table 3: MIMIC III results across frequent (S), few-shot (F), and zero-shot (Z) groups. We mark prior methods for MIMIC datasets that we implemented with a*.

dividual descriptors as input to the GCNN. At test time, we ignore the group codes.

Implementation Details. For the CNN component of our model, we use 300 convolution filters with a filter size of 10 . We use 300 dimensional word embeddings pretrained on PubMed biomedical article titles and abstracts. To avoid overfitting, we use dropout directly after the embedding layer with a rate of 0.2 . For training we use the ADAM (Kingma and Ba, 2015) optimizer with a minibatch size of 8 and a learning rate of 0.001. $q$, the GCNN hidden layer size, is set to 300 . The code for our method is available at https://github.com/bionlproc/ multi-label-zero-shot.

Thresholding has a large influence on traditional multi-label evaluation measures such as micro-F1 and macro-F1 (Tang et al., 2009). Hence, we report both recall at $k(\mathrm{R} @ \mathrm{k})$ and precision at $k$
(P@k) which do not require a specific threshold. $\mathrm{R} @ \mathrm{k}$ is preferred for few- and zero-shot labels, because P@k quickly goes to zero as $k$ increases and gets bigger than the number of group specific labels assigned to each instance. Furthermore, for medical coding, these models are typically used as a recommendation engine to help coders. Unless a label appears at the top of the ranking, the annotator will not see it. Thus, ranking metrics better measure the usefulness of our systems.

Baseline Methods. For the frequent and fewshot labels we compare to state-of-the-art methods on the MIMIC II and MIMIC III datasets including ACNN (Mullenbach et al., 2018) and a CNN method introduced in Baumel et al. (2018). We also compare with the L1 regularized logistic regression model used in Vani et al. (2017). Finally, we compare against our prior EMR coding method, Match-CNN (Rios and Kavuluru, 2018). 


\begin{tabular}{lccc}
\hline & P@ 10 & R@10 & Macro-F1 \\
\hline CNN & 0.562 & 0.407 & 0.028 \\
ACNN & $\mathbf{0 . 6 2 4}$ & $\mathbf{0 . 4 5 2}$ & $\mathbf{0 . 0 6 8}$ \\
Match-CNN & 0.561 & 0.415 & 0.033 \\
ZACNN & 0.577 & 0.429 & 0.037 \\
ZAGCNN & 0.587 & 0.439 & 0.038 \\
\hline
\end{tabular}

Table 4: P@k, R@k, and macro-F1 results over all labels (the union of $\mathrm{S}, \mathrm{F}$, and $\mathrm{Z}$ ).

For zero-shot learning, we compare our results with ESZSL (Romera-Paredes and Torr, 2015). To use ESZSL, we must specify feature vectors for each label. For zero-shot methods, the label vectors used are crucial regardless of the learning method used. Therefore, we evaluate ESZSL with three different sets of label vectors. We average 200 dimensional ICD-9 descriptor word embeddings generated by Pyysalo et al. (2013) which are pretrained on PubMed, Wikipedia, and PubMed Central (ESZSL + W2V). We lowercased descriptors and removed stop-words. We also compare with label vectors derived from our own 300 dimensional embeddings (ESZSL + W2V 2) pretrained on PubMed indexed titles and abstracts. Finally, we generate label vectors using the ICD-9 hierarchy. Specifically, let $\mathbf{Y} \in \mathbb{R}^{N \times L}$ be the document label matrix where $N$ is the total number of documents. We factorize $\mathbf{Y}$ into two matrices $\mathbf{U} \in \mathbb{R}^{N \times 300}$ and $\mathbf{V} \in \mathbb{R}^{300 \times L}$ using graph regularized alternating least squares (GRALS) (Rao et al., 2015). Finally, we also report a baseline using a random ordering on labels, which is important for zero-shot labels - because the total number of such labels is small, the chance that the correct label is in the top $k$ is higher compared to few-shot and frequent labels.

We compare two variants of our method: zeroshot attentive GCNN (ZAGCNN), which is the full method described in Section 3 and a simpler variant without the GCNN layers, zero-shot attentive $\mathrm{CNN}(\mathrm{ZACNN})^{3}$.

Results. Table 2 shows the results for MIMIC II. Because the label set for each medical record is augmented using the ICD-9 hierarchy, we expect methods that use the hierarchy to have an advan-

\footnotetext{
${ }^{3}$ We name our methods with the "zero-shot" prefix because they are primarily designed for such scenarios, although as we show later that these methods are effective for both few-shot and frequent labels
}

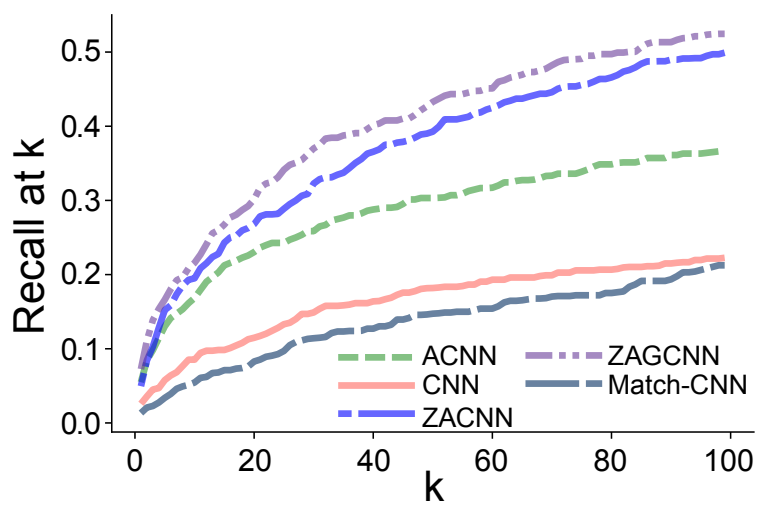

Figure 3: This graph plots the MIMIC III R@k for fewshot $(\mathrm{F})$ labels at different $\mathrm{k}$ values.

tage. Table 2 results do not rely on thresholding because we evaluate using the relative ranking of groups with similar frequencies. ACNN performs best on frequent labels. For few-shot labels, ZAGCNN outperforms ACNN by over 10\% in R@10 and by 8\% in R@5; compared to these R@k gains for few-shot labels, our loss on frequent labels is minimal $(<1 \%)$. We find that the word embedding derived label vectors work best for ESZSL on zero-shot labels. However, this setup is outperformed by GRALS derived label vectors on the frequent and few-shot labels. On zero-shot labels, ZAGCNN outperforms the best ESZSL variant by over16\% for both R@5 and R@10. Also,we find that the GCNN layers help both few- and zeroshot labels. Finally, similar to the setup in Xian et al. (2017), we also compute the harmonic average across all R@5 and all R@10 scores. The metric is only computed for methods that can predict zero-shot classes. We find that ZAGCNN outperforms ZACNN by 4\% for R@ 10.

We report the MIMIC III results in Table 3. Unlike for MIMIC II, the label sets were not expanded using the ICD-9 hierarchy. Yet, we find substantial improvements on both few- and zeroshot labels using a GCNN. ZAGCNN outperforms ACNN by almost $5 \%$ and ZACNN by $1 \%$ in R@10 on few-shot classes. However, ACNN still outperforms all other methods on frequent labels, but by only $0.3 \%$ when compared with ZAGCNN. For zero-shot labels, ZAGCNN outperforms ZACNN by over $5 \%$ and outperforms the best ESZSL method by nearly $20 \%$ in R@ 10 . We find that ZACNN slightly underperforms ZAGCNN on frequent labels with more prominent differences showing up for infrequent labels.

In Table 4 we compare the P@10, R@10, and 
macro-F1 measures across all three groups (the union of $S, F$, and $Z$ ) on the MIMIC III dataset. We emphasize that the evaluation metrics are calculated over all labels and are not averages of the metrics computed independently for each group. We find that R@10 is nearly equivalent to the $\mathrm{R} @ 10$ on the frequent group in Table 3. Furthermore, we find that ACNN outperforms ZAGCNN in P@10 by almost 4\%. To compare all methods with respect to macro-F1, we simply threshold each label at 0.5. Both R@k and P@k give more weight to frequent labels, thus it is expected that ACNN outperforms ZAGCNN for frequent labels. However, we also find that ACNN outperforms our methods with respect to Macro-F1.

Given macro-F1 equally weights all labels, does the higher macro score mean ACNN performs better across infrequent labels? In Figure 3, we plot the MIMIC III R@k for the neural methods with $k$ ranging from 1 to 100 . We find as $k$ increases, the differences between ZAGCNN and ACNN become more evident. Given Figure 3 and the scores in Table 3, it is clear that ACNN does not perform better than ZAGCNN with respect to fewand zero-shot labels. The improvement in macro$\mathrm{F} 1$ for ACNN is because it performs better on frequent labels. In general, infrequent labels will have scores much less than 0.5. If we rank all labels ( $S \cup F \cup Z$ ), we find that few-shot labels only occur among the top 16 ranked labels (average number of labels for MIMIC III) for $6 \%$ of the test documents that contain them. This suggests that many frequent irrelevant labels have higher scores than the correct few-shot label.

Why do the rankings among few- and zero-shot labels matter if they are rarely ranked above irrelevant frequent labels? If we can predict which instances contain infrequent labels (novelty detection), then we can help human coders by providing them with multiple recommendation lists - a list of frequent labels and a list of infrequent/zeroshot labels. Also, while we would ideally want a single method that performs best for both frequent and infrequent labels, currently we find that there is a trade-off between them. Hence it may be reasonable to use different methods in combination depending on label frequency.

\section{Conclusion and Future Work}

In this paper, we performed a fine-grained evaluation of few- and zero-shot label learning in the large-scale multi-label setting. We also introduced a neural architecture that incorporates label descriptors and the hierarchical structure of the label spaces for few- and zero-shot prediction. For these infrequent labels, previous evaluation methodologies do not provide a clear picture about what works. By evaluating power-law datasets using a generalized zero-shot learning methodology, we provide a staring point toward a better understanding. Our proposed architecture also provides large improvements on infrequent labels over state-ofthe-art automatic medical coding methods.

We believe there are two important avenues for future work.

1. For medical coding, a wealth of unstructured domain expertise is available in biomedical research articles indexed by PubMed. These articles are annotated with medical subject headings (MeSH terms), which are organized in a hierarchy. Relationships between $\mathrm{MeSH}$ terms and ICD-9 codes are available in Unified Medical Language System (UMLS (Bodenreider, 2004)). If we can take advantage of all this structured and unstructured information via methods such as transfer learning or multi-task learning, then we may be able to predict infrequent labels better.

2. For our method to be useful for human coders, it is important to develop an accurate novelty detector. We plan to study methods for determining if an instance contains an infrequent label and if it does, how many infrequent labels it should be annotated with. In essence, this is an extension of the MetaLabeler (Tang et al., 2009) methodology and open classification (Shu et al., 2017). If we can predict if an instance contains infrequent labels, then we can recommend few- and zero-shot labels only when necessary.

\section{Acknowledgements}

Thanks to the outstanding reviewers who provided invaluable insights to improve our manuscript. This research is supported by the U.S. National Library of Medicine through grant R21LM012274. We also gratefully acknowledge the support of the NVIDIA Corporation for its donation of the Titan $\mathrm{X}$ Pascal GPU used for this research. 


\section{References}

Zeynep Akata, Florent Perronnin, Zaid Harchaoui, and Cordelia Schmid. 2016. Label-embedding for image classification. IEEE transactions on pattern analysis and machine intelligence, 38(7):1425-1438.

Miltiadis Allamanis, Hao Peng, and Charles Sutton. 2016. A convolutional attention network for extreme summarization of source code. In International Conference on Machine Learning, pages 2091-2100.

Joost Bastings, Ivan Titov, Wilker Aziz, Diego Marcheggiani, and Khalil Simaan. 2017. Graph convolutional encoders for syntax-aware neural machine translation. In Conference on Empirical Methods in Natural Language Processing, pages $1957-$ 1967.

Tal Baumel, Jumana Nassour-Kassis, Michael Elhadad, and Noemie Elhadad. 2018. Multi-label classification of patient notes a case study on icd code assignment. In AAAI Joint Workshop on Health Intelligence.

Kush Bhatia, Himanshu Jain, Purushottam Kar, Manik Varma, and Prateek Jain. 2015. Sparse local embeddings for extreme multi-label classification. In $\mathrm{Ad}$ vances in Neural Information Processing Systems, pages $730-738$.

Olivier Bodenreider. 2004. The unified medical language system (umls): integrating biomedical terminology. Nucleic acids research, 32(suppl_1):D267D270.

Meihao Chen, Zhuoru Lin, and Kyunghyun Cho. 2017. Graph convolutional networks for classification with a structured label space. arXiv preprint arXiv:1710.04908.

Michaël Defferrard, Xavier Bresson, and Pierre Vandergheynst. 2016. Convolutional neural networks on graphs with fast localized spectral filtering. In $A d$ vances in Neural Information Processing Systems, pages 3844-3852.

Alistair EW Johnson, Tom J Pollard, Lu Shen, Liwei H Lehman, Mengling Feng, Mohammad Ghassemi, Benjamin Moody, Peter Szolovits, Leo Anthony Celi, and Roger G Mark. 2016. MIMIC-III, a freely accessible critical care database. Scientific data, 3 .

V Jouhet, G Defossez, A Burgun, P le Beux, P Levillain, P Ingrand, and V Claveau. 2012. Automated classification of free-text pathology reports for registration of incident cases of cancer. Methods of Information in Medicine, 51(3):242-251.

Nal Kalchbrenner, Edward Grefenstette, and Phil Blunsom. 2014. A convolutional neural network for modeling sentences. In Annual Meeting of the Association for Computational Linguistics (ACL), pages 655-665.
Yoon Kim. 2014. Convolutional neural networks for sentence classification. In Conference on Empirical Methods in Natural Language Processing (EMNLP), pages 1746-1751.

Diederik P. Kingma and Jimmy Ba. 2015. Adam: A method for stochastic optimization. In International Conference on Learning Representations (ICLR).

Thomas N. Kipf and Max Welling. 2017. Semisupervised classification with graph convolutional networks. In International Conference on Learning Representations (ICLR).

Gregory Koch, Richard Zemel, and Ruslan Salakhutdinov. 2015. Siamese neural networks for one-shot image recognition. In ICML Deep Learning Workshop, volume 2 .

Diego Marcheggiani and Ivan Titov. 2017. Encoding sentences with graph convolutional networks for semantic role labeling. In Conference on Empirical Methods in Natural Language Processing (EMNLP), pages 1506-1515.

Thomas Mensink, Efstratios Gavves, and Cees GM Snoek. 2014. Costa: Co-occurrence statistics for zero-shot classification. In IEEE Conference on Computer Vision and Pattern Recognition (CVPR), pages 2441-2448.

Tomas Mikolov, Andrea Frome, Samy Bengio, Jonathon Shlens, Yoram Singer, Greg S Corrado, Jeffrey Dean, and Mohammad Norouzi. 2013. Zeroshot learning by convex combination of semantic embeddings. In International Conference on Learning Representations (ICLR).

James Mullenbach, Sarah Wiegreffe, Jon Duke, Jimeng Sun, and Jacob Eisenstein. 2018. Explainable prediction of medical codes from clinical text. In North American Chapter of the Association for Computational Linguistics (NAACL).

Vinod Nair and Geoffrey E Hinton. 2010. Rectified linear units improve restricted Boltzmann machines. In International Conference on Machine Learning (ICML), pages 807-814.

Jinseok Nam, Jungi Kim, Eneldo Loza Mencía, Iryna Gurevych, and Johannes Fürnkranz. 2014. Largescale multi-label text classification - revisiting neural networks. In Machine Learning and Knowledge Discovery in Databases, ECML PKDD, pages 437452.

NCHS. 1978. International classification of diseases, ninth revision, clinical modification (icd-9-cm). $\mathrm{Hy}$ attsville (MD): National Center for Health Statistics.

Yannis Papanikolaou, Grigorios Tsoumakas, Manos Laliotis, Nikos Markantonatos, and Ioannis P Vlahavas. 2015. Auth-atypon at bioasq 3: Large-scale semantic indexing in biomedicine. In CLEF (Working Notes). 
Ioannis Partalas, Aris Kosmopoulos, Nicolas Baskiotis, Thierry Artières, George Paliouras, Éric Gaussier, Ion Androutsopoulos, Massih-Reza Amini, and Patrick Gallinari. 2015. LSHTC: A benchmark for large-scale text classification. CoRR, abs/1503.08581.

Hao Peng, Jianxin Li, Yu He, Yaopeng Liu, Mengjiao Bao, Lihong Wang, Yangqiu Song, and Qiang Yang. 2018. Large-scale hierarchical text classification with recursively regularized deep graph-cnn. In World Wide Web Conference on World Wide Web, pages 1063-1072.

Adler Perotte, Rimma Pivovarov, Karthik Natarajan, Nicole Weiskopf, Frank Wood, and Noémie Elhadad. 2013. Diagnosis code assignment: models and evaluation metrics. Journal of the American Medical Informatics Association, 21(2):231-237.

S. Pyysalo, F. Ginter, H. Moen, T. Salakoski, and S. Ananiadou. 2013. Distributional semantics resources for biomedical text processing. In $L B M$, pages 39-44.

Nikhil Rao, Hsiang-Fu Yu, Pradeep K Ravikumar, and Inderjit S Dhillon. 2015. Collaborative filtering with graph information: Consistency and scalable methods. In Advances in neural information processing systems, pages 2107-2115.

A Rios and R Kavuluru. 2015. Analyzing the moving parts of a large-scale multi-label text classification pipeline: Experiences in indexing biomedical articles. In IEEE International Conference on Healthcare Informatics, volume 2015, pages 1-7.

Anthony Rios and Ramakanth Kavuluru. 2018. Emr coding with semi-parametric multi-head matching networks. In North American Chapter of the Association for Computational Linguistics (NAACL), volume 1, pages 2081-2091.

Bernardino Romera-Paredes and Philip Torr. 2015. An embarrassingly simple approach to zero-shot learning. In International Conference on Machine Learning, pages 2152-2161.

Timothy N Rubin, America Chambers, Padhraic Smyth, and Mark Steyvers. 2012. Statistical topic models for multi-label document classification. $M a$ chine Learning, 88(1-2):157-208.

Ubai Sandouk and Ke Chen. 2016. Multi-label zeroshot learning via concept embedding. arXiv preprint arXiv:1606.00282.

Michael Schlichtkrull, Thomas N Kipf, Peter Bloem, Rianne van den Berg, Ivan Titov, and Max Welling. 2018. Modeling relational data with graph convolutional networks. In Proceedings of 15th European Semantic Web Conference (ESWC 2018).

Lei Shu, Hu Xu, and Bing Liu. 2017. Doc: Deep open classification of text documents. In Proceedings of the 2017 Conference on Empirical Methods in Natural Language Processing, pages 2911-2916.
Jake Snell, Kevin Swersky, and Richard Zemel. 2017. Prototypical networks for few-shot learning. In $A d$ vances in Neural Information Processing Systems, pages 4080-4090.

Emma Strubell and Andrew McCallum. 2017. Dependency parsing with dilated iterated graph CNNs. In Workshop on Structured Prediction for Natural Language Processing, SPNLP@EMNLP, pages 1-6.

Lei Tang, Suju Rajan, and Vijay K Narayanan. 2009. Large scale multi-label classification via metalabeler. In Proceedings of the 18th international conference on World wide web, pages 211-220. ACM.

George Tsatsaronis, Michael Schroeder, Georgios Paliouras, Yannis Almirantis, Ion Androutsopoulos, Eric Gaussier, Patrick Gallinari, Thierry Artieres, Michael R Alvers, Matthias Zschunke, et al. 2012. Bioasq: A challenge on large-scale biomedical semantic indexing and question answering. In $A A A I$ fall symposium: Information retrieval and knowledge discovery in biomedical text.

Grigorios Tsoumakas, Ioannis Katakis, and Ioannis P. Vlahavas. 2010. Mining multi-label data. In Data Mining and Knowledge Discovery Handbook, pages 667-685.

Ankit Vani, Yacine Jernite, and David Sontag. 2017. Grounded recurrent neural networks. arXiv preprint arXiv:1705.08557.

Oriol Vinyals, Charles Blundell, Tim Lillicrap, Daan Wierstra, et al. 2016. Matching networks for one shot learning. In Advances in Neural Information Processing Systems, pages 3630-3638.

Xiaolong Wang, Yufei Ye, and Abhinav Gupta. 2018. Zero-shot recognition via semantic embeddings and knowledge graphs. arXiv preprint arXiv:1803.08035.

Jason Weston, Sumit Chopra, and Keith Adams. 2014. \# tagspace: Semantic embeddings from hashtags. In Conference on Empirical Methods in Natural Language Processing (EMNLP), pages 1822-1827.

Yongqin Xian, Bernt Schiele, and Zeynep Akata. 2017. Zero-shot learning-the good, the bad and the ugly. In IEEE Conference on Computer Vision and Pattern Recognition, pages 3077-3086. IEEE Computer Society.

Zichao Yang, Diyi Yang, Chris Dyer, Xiaodong He, Alex Smola, and Eduard Hovy. 2016. Hierarchical attention networks for document classification. In North American Chapter of the Association for Computational Linguistics (NAACL), pages 14801489.

Hsiang-Fu Yu, Prateek Jain, Purushottam Kar, and Inderjit S. Dhillon. 2014. Large-scale multi-label learning with missing labels. In Proceedings of the 31th International Conference on Machine Learning, pages 593-601. 